\title{
A Small-Angle X-Ray Scattering Instrument Equipped With The Imaging Plate
}

\author{
Tomoaki Kamiyama and Kenji Suzuki \\ Institute for,Materials Research, Tohoku University, \\ 2-1-1 Katahira, Aobaku, Sendai 980, Japan
}

(Received August 15, 1997; final form August 29, 1997)

\begin{abstract}
A pinhole small-angle X-ray scattering (SAXS) instrument was designed by coupling with the imaging plate. The details of the apparatus including data processing were described. The capability of this new SAXS instrument was demonstrated by measuring colloidal silica suspensions.
\end{abstract}

\section{INTRODUCTION}

Small-angle scattering (SAS) of X-rays (SAXS) and neutrons (SANS) is a widely used diffraction method for studying the structure of matter $/ 1 /$. The use of $\mathrm{X}$ rays and neutrons, with wavelengths of about $1 \AA$, permits the study of the atomic structure on a length scale from $10 \AA$ up to thousands of angstroms which contributes appreciably to the diffraction pattern in the small angle region. The atomic structure on the same length scale can be observed directly by transmission electron microscopy (TEM). Small-angle scattering techniques have the advantages of permitting us to carry out structural investigation of a sample in a liquid state like a colloidal suspension and to determine physical quantities being spatially averaged over all the sample volume irradiated with the incident beam.

A pinhole camera has become feasible in combination with the use of a powerful X-ray source and a two-dimensional position-sensitive detector. The employment of a pinhole camera permits us the direct observation of the anisotropic scattering patterns from the entities such as fibers as shown in Ref, $/ 2 /$. Circular average of two-dimensional data could lead to an increase in the signal-to-noise ratio of scattered intensity by orders of magnitude, especially in the Porod region.

In this paper we will describe the details of a pinhole SAXS instrument using an imaging plate and the application of the SAXS instrument to studies of the structure and interactions between particles in silica sols with various silica concentrations.

\section{SMALL-ANGLE X-RAY SCATTERING}

2.1. The overall features of the small-angle X-ray scattering instrument

The principal scheme and the overall structure of the small-angle $x$-ray instrument are illustrated in Fig. 1 and Fig. 2, respectively. the instrument is composed mainly of a radiation source, a collimator of the beam, a specimen block and a detector. An incident X-ray beam is formed into a $0.5 \times 0.5 \mathrm{~mm}$ size by a set of two apertures, a source aperture and a defining aperture, and the parasitic scattering mainly from the edges of the defining (second) aperture is shielded by a guard (third) aperture. The sample is placed just after the guard aperture. The direct beam is blocked by a beamstop in front of the detector.

An X-ray generator and a monochromator are mounted on a goniometer. All the other instrumental components, such as the collimation systems, the specimen chamber and the imaging plate chamber, are mounted respectively on two rails of $3500 \mathrm{~mm}$ length. Every block can be moved to new positions readily. 


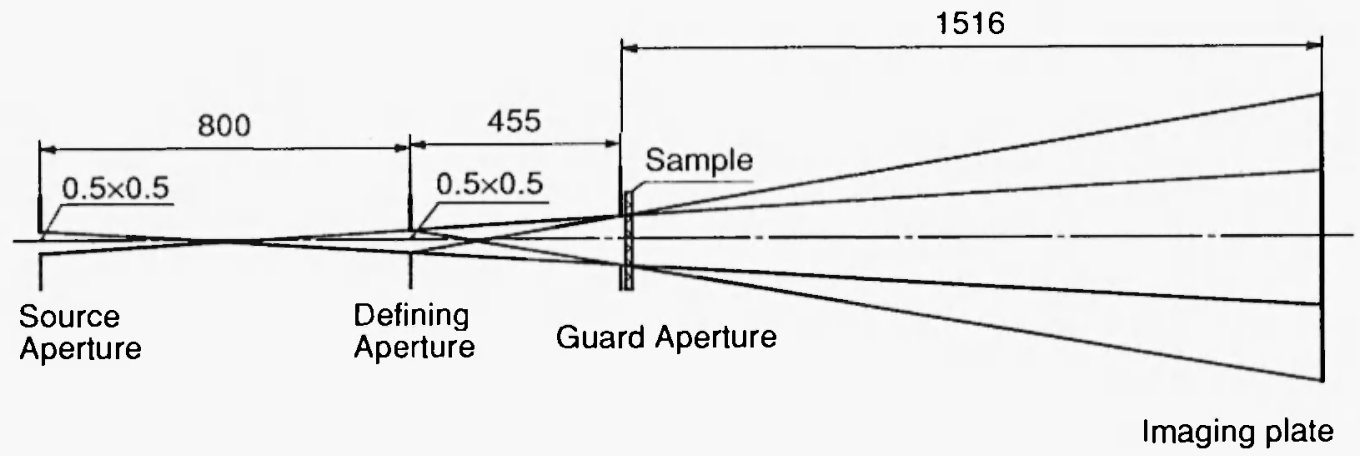

Fig. 1: The principles of a small-angle X-ray scattering instrument.
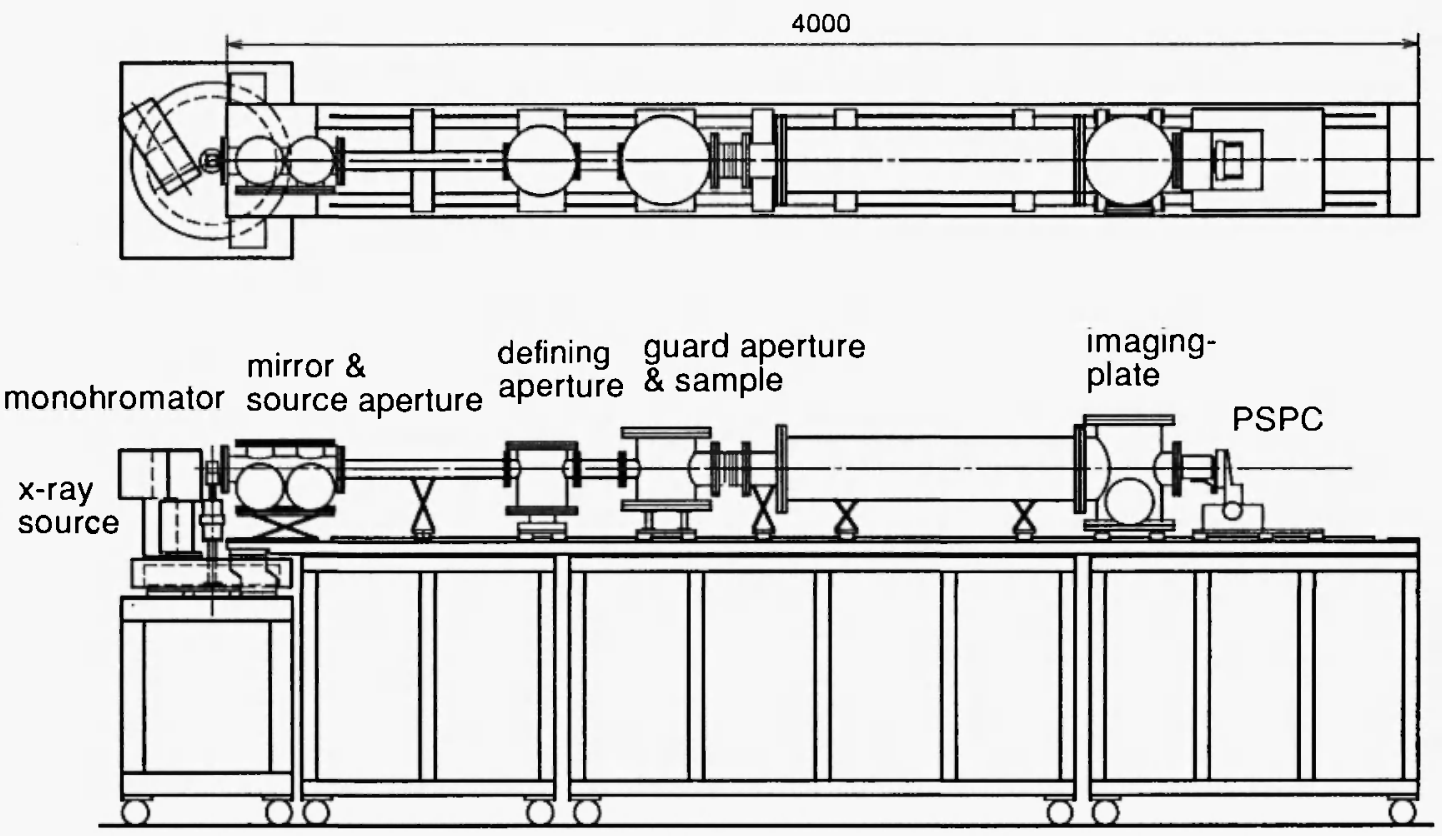

Fig. 2: Schematic representations of the small-angle X-ray scattering instrument.

With this setup, the geometry of the spectrometer can be changed readily, in accordance with a length scale of structured inhomogeneities to be measured.

A crystal monochromator and a mirror are located outside the collimation system, and any device that may become a noise-irradiator is not located along the evacuated beam path between the source and defining apertures. The atmosphere along the beam path gives rise to not only a decrease in the incident $\mathrm{X}$-ray beam intensity by the absorption but also the strong lowangle scattering from the atmospheric molecules. All the X-ray flight path including the sample and detector chambers is evacuated to pressure $<10^{-2}$ Torr by a rotary pump, which is expected to lead to an increase in a signal-to-noise ratio of the camera.

2.2. Instrumental components of the small-angle $X$ ray scattering camera

A $18 \mathrm{~kW}$ rotating-anode $\mathrm{X}$-ray generator, ultraX 18 (Rigaku-Denki), is mounted on a goniometer as illustrated in Fig. 3. The goniometer is composed of two 


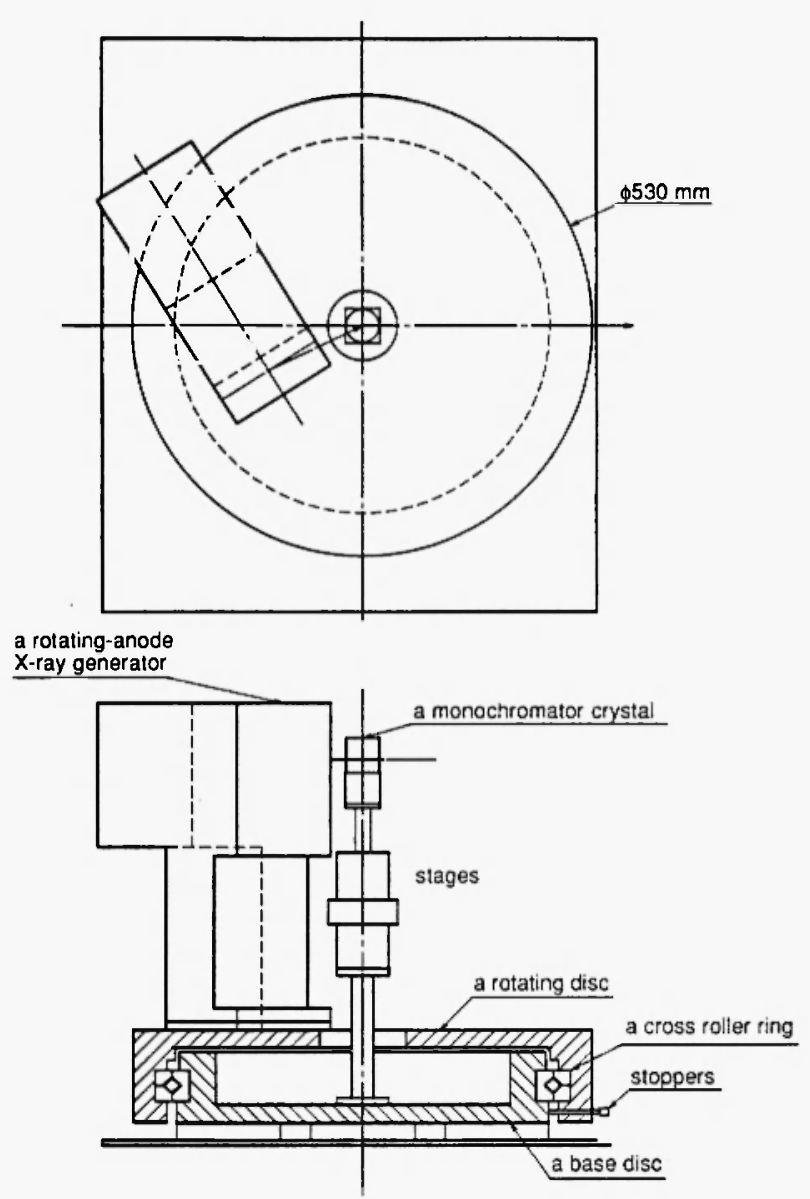

Fig. 3: Goniometer of the X-ray source.

discs connected by a cross roller ring. The upper disc on which the rotating-anode $\mathrm{X}$-ray source is fixed can pivot about the monochromator crystal which is mounted through a small goniometer at the center of the fixed bottom disc. Monochromatization is done by Bragg scattering by using a small piece of pyrolytic graphite (Panasonic graphite PGC-X03, d = $3.354 \AA$ and a $0.3^{\circ} \sim 0.4^{\circ}$ mosaic spread, Matsushita Electric Industrial Co., Ltd.).

The higher-order contamination of the incident beam passed by the monochromator can be cut offiby a mirror using the total reflection effect of X-rays. A fused silica mirror coated with Pt plating of $50 \mathrm{~nm}$ thickness whose size is $150 \times 30 \mathrm{~mm}$ and the radius of curvature is 56 meters (SIGMA KOKI Co., Ltd.) is used. This cut-off is very important in an X-ray detection system using the imaging plate, because the output signal of the imaging plate depends not only on the number of the collected X-ray photons but also on the wave length of the photon. The higher-order contamination of the incident beam passed by a monochromator must be eliminated.

Collimating two apertures, a source and a defining aperture, consist of sets of four tantalum sheets $0.3 \mathrm{~mm}$ in thickness mounted to make $0.5 \mathrm{~mm}$ square apertures. The polished edges of the sheets had surfaces approximately parallel to the incident beam cut to make a $3^{\circ}$ taper, to reduce parasitic scattering originating from scattering at the edges /3/. A guard (third) aperture is located just before a sample position. The third aperture also consists of the four tantalum sheets and the diameter of the third aperture is adjusted so that the direct beam will not touch it.

The principles and performance of the imaging plate detection system have been described exactly in many papers (see for example /4/). The imaging plate for X-ray detection in the SAXS instrument is $125 \times$ $200 \mathrm{~mm}$ in size and is fixed on a cassette by a beam stop (Fig. 4). This permits measurements to be free from any correction for the shadowing effect of an arm supporting the beam stop. The location of the cassette can be reproduced within an accuracy of $0.01 \mathrm{~mm}$ in both directions by attaching the cassette to an $x-y$ stage as shown in Fig. 4. The cassette must be located at the same position with an accuracy within the pixel size for one set of measurements.

The settling of the imaging plate in an evacuated chamber makes it unnecessary to use the large size exit window, $125 \times 200 \mathrm{~mm}$ in this case. The absorption of the scattered X-rays by the exit window can be avoided. Further, this settling promises SAXS measurements free from any correction for the non-uniformity of the thickness of the window and the shadowing effect of the grid supporting the exit window.

\subsection{Manipulation and reduction of two-dimensional data}

A stored image is read out from the imaging plate manually mounted on the drum of an image read-out system R-AXIS DS3A (Rigaku-Denki) and is transferred into an array of binary data of $1900 \times 1150$ pixels whose pixel size is $100 \times 100 \mu \mathrm{m}$. The twodimensional data collected on the read-out system are transmitted to a UNIX workstation for manipulation 


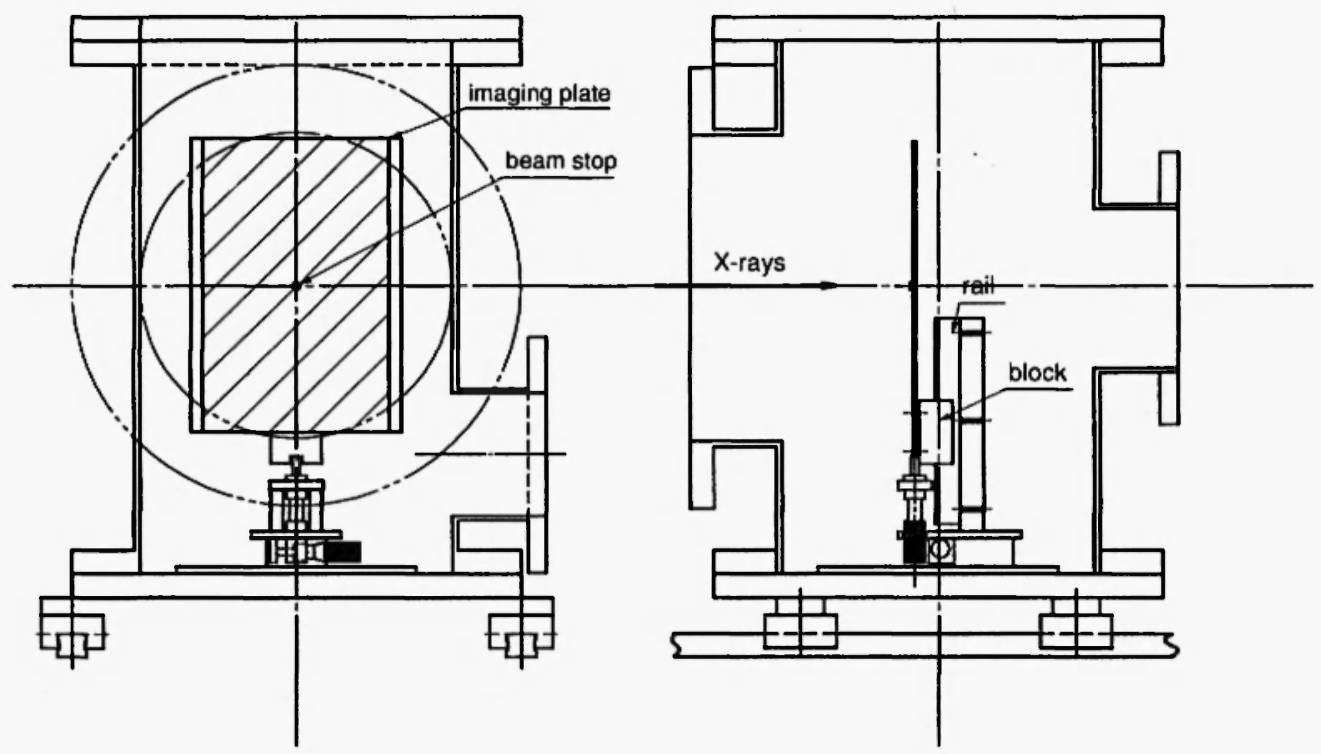

Fig. 4: Imaging-plate chamber.

and display. Software routines for the manipulation and display of the two-dimensional data on the HP workstation have been developed using the $\mathbf{C}$ programming language with the help of the Graphic Kernel System NOVA*GKS (Nova Graphics International Corporation $/ 5 /$ ) for a two-dimensional graphic display. As easily noticed, the data acquisition is done in an off-line mode and the scattering pattern cannot be observed during the measurements.

There is no advantage to having the resolution of the detecting system much better than the width of the incident beam at the beam stop $/ 6 /$. Accordingly, the pixel size $0.1 \times 0.1 \mathrm{~mm}$ of output data is considered to have no advantage in the data collection on this pinhole geometry SAXS instrument of the incident beam $0.5 \times$ $0.5 \mathrm{~mm}$ in size. The pixel size $0.5 \times 0.5 \mathrm{~mm}$ is used for the manipulation and display of data, by reducing a $1900 \times 1150$ array data to that of a $380 \times 230$ array. This transformation could reduce the size of a computer memory required for the storage and manipulation of two-dimensional data and could result in rapid operation for data manipulation and display on the screen. Furthermore, the transformation makes effective resolution of the detecting system to be determined not by the resolution function but by the pixel size of the imaging plate. The spatial resolution of the imaging plate is less than $0.2 \mathrm{~mm} / 4 /$.

The data reduction of two-dimensional data to one- dimensional data depending only on a scalar $q$ is performed by the radial or sector average of the data of a $380 \times 230$ array around the beam center. The radial or sector average $X_{i}$ of scattered intensities is performed over the pixels at a distance $r_{i}$ from the beam center. The modulus of the scattering vector $q_{1}$ is calculated from

$\mathrm{q}_{\mathrm{i}}=\frac{4 \pi \sin \theta_{i}}{\lambda}$,

where the scattering angle $2 \theta_{\mathrm{i}}$ is obtained from

$2 \theta_{i}=\tan ^{-1}\left(r_{i} / d\right)$

The sample-detector distance $d$ is determined from the Bragg spacing of a standard sample in the same geometry of the detecting system as sample measurements. The averaged intensity $X_{i}$, the modulus of the scattering vector $q$, and the square root of the variance $\mathrm{E}\left(\mathrm{X}_{\mathrm{i}}^{2}\right)$ are stored in i-th memory location.

\subsection{Resolution of the instrument}

The angular resolution $\mathrm{R}$ can be calculated from the following expression $/ 2$ :

$R=\alpha+\beta$,

where $\alpha=\left(a_{1}+a_{1}\right) / 2 L_{1}$, and $\beta=\left(a_{2}+a_{3}\right) / 2 L_{2}$ for a 
collimation system with two square pinholes of the same size, $a_{1} \times a_{1}$, separated by a distance $L_{1}$ and a detector with a resolving element of size $a_{3} \times a_{3}$ separated by a distance $L_{2}$ from a specimen of beamirradiated area $a_{2} \times 22 . \alpha=6.25 \times 10^{-4}$ and $\beta=5.27 \times$ $10^{-4}$ are calculated for pinhole geometry as illustrated in Fig. 1 and $R=1.15 \times 10^{-3}$ is obtained from equation (3). The uncertainty $\Delta q$ due to this angular resolution $R$ is $4.69 \times 10^{-3} \AA^{-1}$.

In combination with the collimation system described above, the graphite crystal of a mosaic spread of $0.3^{\circ} \sim 0.4^{\circ}$ permits the wavelength resolution about $1 \%$. Therefore, the $\mathrm{Cu}-\mathrm{K} \alpha_{1}$ and $\mathrm{K} \alpha_{2}$ radiation with wavelengths $1.537395 \AA$ and $1.541232 \AA$ respectively, are allowed to pass. The uncertainty $\triangle M \lambda$ is considered due to the difference between the wavelengths of $\mathrm{Cu}$ $K \alpha_{1}$ and $K \alpha_{2}$ radiation and $2.49 \times 10^{-3}$ is obtained. The corresponding $\Delta \mathrm{q}$ is estimated to be $2.5 \times 10^{-4} \AA^{-1}$ at $\mathrm{q}$ $=0.1 \AA^{1}$.

The total uncertainty becomes $\Delta \mathrm{q}=4.94 \times 10^{-3} \AA^{-1}$. Fig. 5(a) shows the isointensity scattering pattern from bovine collagen measured for 30 minutes. The scattered
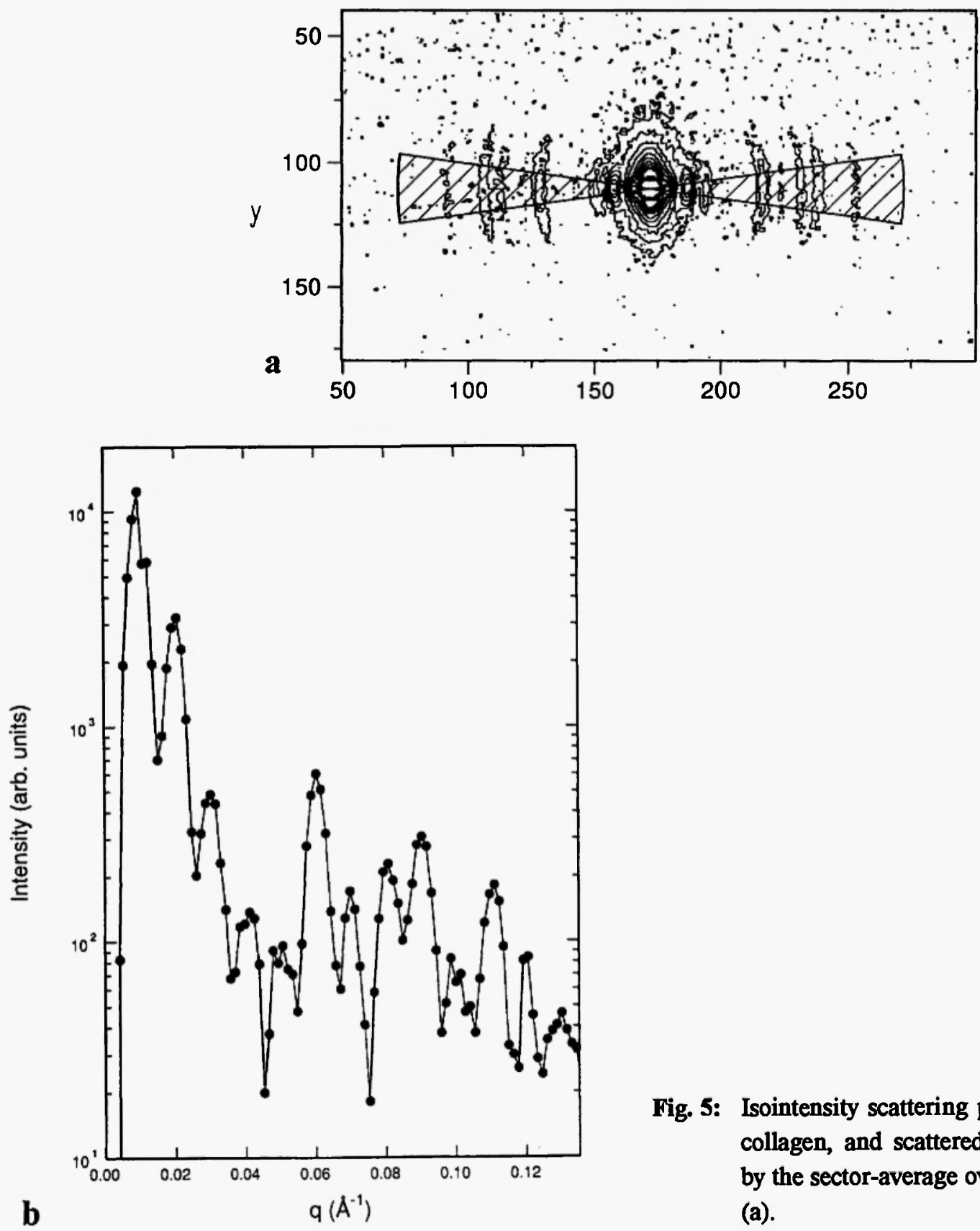

Fig. 5: Isointensity scattering pattern (a) from bovine collagen, and scattered intensity (b) obtained by the sector-average over the hatched areas in (a). 
intensity versus the modulus of the scattering vector averaged over the sector regions specified in the figure is plotted in Fig. 5(b). The d-spacing obtained from the six-order peak is $625 \AA$. Twelve peaks are clearly identified. The uncertainty $\Delta \mathrm{q}_{\mathrm{FWFM}} \approx 5.5 \times 10^{-3} \AA^{-1}$ is estimated from the measured peaks.

\subsection{The smearing effect of the instrument}

Infinitesimally small (ideal) pinholes lead to no smearing effects on the scattered intensity. In the actual measurement, however, the collimation smearing effect arises from the finite size of the collimation apertures and the finite pixel size of two-dimensional data. The measured scattering curve is obtained by averaging the real scattering data over an appreciable range around the nominal scattering angle and possibly becomes different from the curve for perfect collimation. Accordingly, it is very important to take into account 'smearing' of the ideal curve profile as a result of the finite dimensions of the beam, the spatial resolution of the detector and the polychromaticity of the radiation.

The monochromatizing system would allow both the $\mathrm{Cu}-\mathrm{K} \alpha_{1}$ and $\mathrm{K} \alpha_{2}$ radiations to pass. However, the uncertainty $\Delta q$ arising from the difference of the wavelengths of $\mathrm{Cu}-\mathrm{K} \alpha_{1}$ and $\mathrm{K} \alpha_{2}$ radiations is less than one tenth of the uncertainty arising from the angular resolution of the collimation system. The distortion due to the polychromaticity of the radiation can be neglected in this case.

Collimation effects can be described by the 'weighting function', which specifies the width of the interval over which the intensity is averaged and indicates the emphasis given to scattering angles within this interval $7 /$. An ideal scattering function I(q) can be related to the scattered intensity observed in a real experiment, $\mathrm{I}_{\mathrm{obr}}(\mathrm{q})$, through equation

$I_{\text {obe }}(q)=\int I\left(q-q^{\prime}\right) W\left(q, q^{\prime}\right) d q^{\prime}$,

where $W$ is the weighting function. In Moore's expansion technique, an ideal scattering function $I(q)$ is expressed as $/ 81$

$I\left(q_{i}\right)=4 \sum_{n=1}^{\infty} a_{n} \frac{B(n, i)}{q_{i}}, \quad$ and
$B(n, i)=\frac{2 \pi^{2} n d(-1)^{n+i} \sin \left(q_{i} d\right)}{(\pi n)^{2}-\left(q_{i} d\right)^{2}}$.

The radial average of the two-dimensional profile of the incident beam recorded on the detector plane was used for the weighting function $W$. The length $d=240 \AA$ in Eq. (5) is chosen to be as small as possible while not being smaller than the largest correlation length in the sample. The coefficients $a_{n}$ were determined by using a weighted least-squares method for the scattered intensity of $0.95 \mathrm{wt} . \%$ Ludox colloidal solution, $\mathrm{I}_{\mathrm{ob}}(\mathrm{q})$, in the $q$ range between $0.008 \AA^{-1}$ and $0.15 \AA^{-1}$.

Fig. 6 shows the result of the Moore's expansion technique. The effect of collimation smearing on the experimental data is small in the region between $q=$ $0.01 \AA^{-1}$ and $0.1 \AA^{-1}$ where the scattered intensity with a good $\mathrm{S} / \mathrm{N}$ ratio is measured. The smearing effects in the pinhole small-angle $\mathrm{X}$-ray scattering instrument are

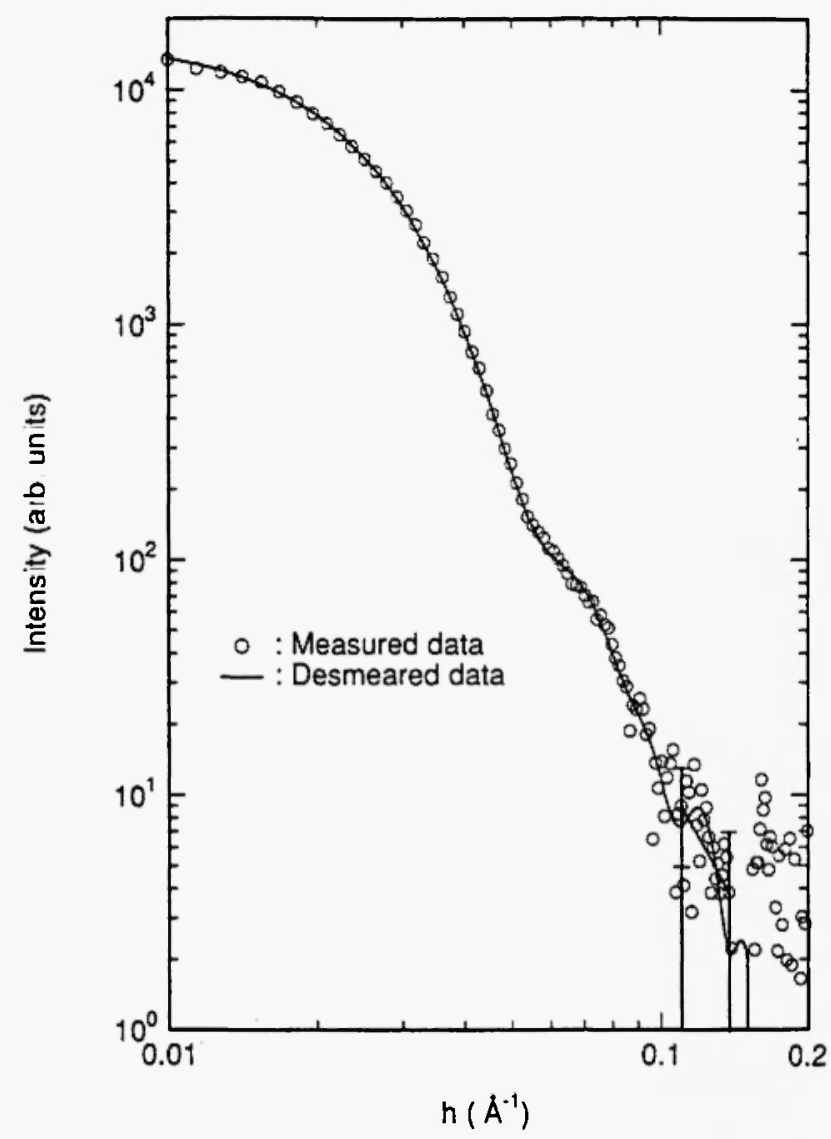

Fig. 6: Results of the desmearing process of Moore's technique for 0.95 wt.\% silica colloidal suspension. 
negligible and the raw data $\mathrm{I}_{\mathrm{obs}}(\mathrm{q})$ can be used for further analysis without smearing.

\subsection{Data analysis}

In scattering experiments the scattered intensity $I(q)$ from a specimen of thickness $t$ and linear absorption $\mu$ is evaluated from the scattered power $P(q)$ recorded in a detector resolving element at the pixel corresponding to the scattering vector $q$ using an expression (e.g., /6,9):

$P(q)=I_{0} t \exp (-\mu t) I(q) \Delta \Omega$,

where $\mathrm{I}_{0}$ is the incident-beam intensity and $\Delta \Omega$ is the solid angle subtended by the detector resolving element (steradian).

The differential scattering cross section per unit volume of a specimen $I(q)$ can be calculated from Eq. (6). This calculation requires the evaluation of $\mathrm{I}_{0}, \Delta \Omega$, the transmission coefficient $\exp (-\mu t)$ and thickness $t$ of a specimen. The transmission coefficient of a specimen $\exp (-\mu t)$ can be determined with high precision by measuring the attenuation of the scattering power from a strong scatterer by inserting the specimen across the beam line between the collimating apertures in this SAXS instrument. The measurement of the primary beam intensity $\mathrm{I}_{0}$ is not straightforward. It should be noted here that the primary beam intensity $I_{0}$ evaluated using the imaging plate is not equal to the exact number of the incident photons but proportional to it. The output signal from the imaging-plate reader depends not only on the energy of incident photons but also on a voltage supplied to a photomultiplier.

In this study, the product $\mathrm{I}_{0} \Delta \Omega=2.24 \mathrm{sec}^{-1}$ for $\mathrm{Cu}$ $\mathrm{K} \alpha$ radiation under the operating conditions of $40 \mathrm{kV}$ and $300 \mathrm{~mA}$ was obtained from the SAXS measurement of silica colloidal suspension with a known silica concentration of $0.950 \mathrm{wt} . \%$, using equation

$\frac{1}{I_{0} \Delta \Omega} \int_{0}^{\infty} q^{2} P(q) d q=2 \pi^{2} I_{e} \varphi_{1} \varphi_{2}\left(\rho_{1}-\rho_{2}\right)$,

which is given in terms of the volume fraction of the suspended silica particles, $\varphi_{1}$, that of the suspension fluid water, $\Phi_{2}$, and corresponding electron densities, $\rho_{1}$ and $\rho_{2}$, where $I_{0}=7.94 \times 10^{-26} \mathrm{~cm}^{2}$.

The measured data of the Lupolen standard was converted into absolute units using the calibration factor $I_{0} \Delta \Omega$. The result shows that the scattered intensity of the Lupolen standard exhibits a broad reflection with a peak height $6.07 \mathrm{~cm}^{-1}$ at $0.027 \AA^{-1}$ (Fig. 7). According to T.P. Russell et al. /10., three separate SAXS measurements of the Lupolen standard were made and determined the peak intensity near $0.03 \AA^{-1}$ to be 5.3 (ORNL $10 \mathrm{~m} \mathrm{SAXS),} 5.03$ (SSRL) and 6.0 $\mathrm{cm}^{-1}$ (Schelten and Hendricks /6/).

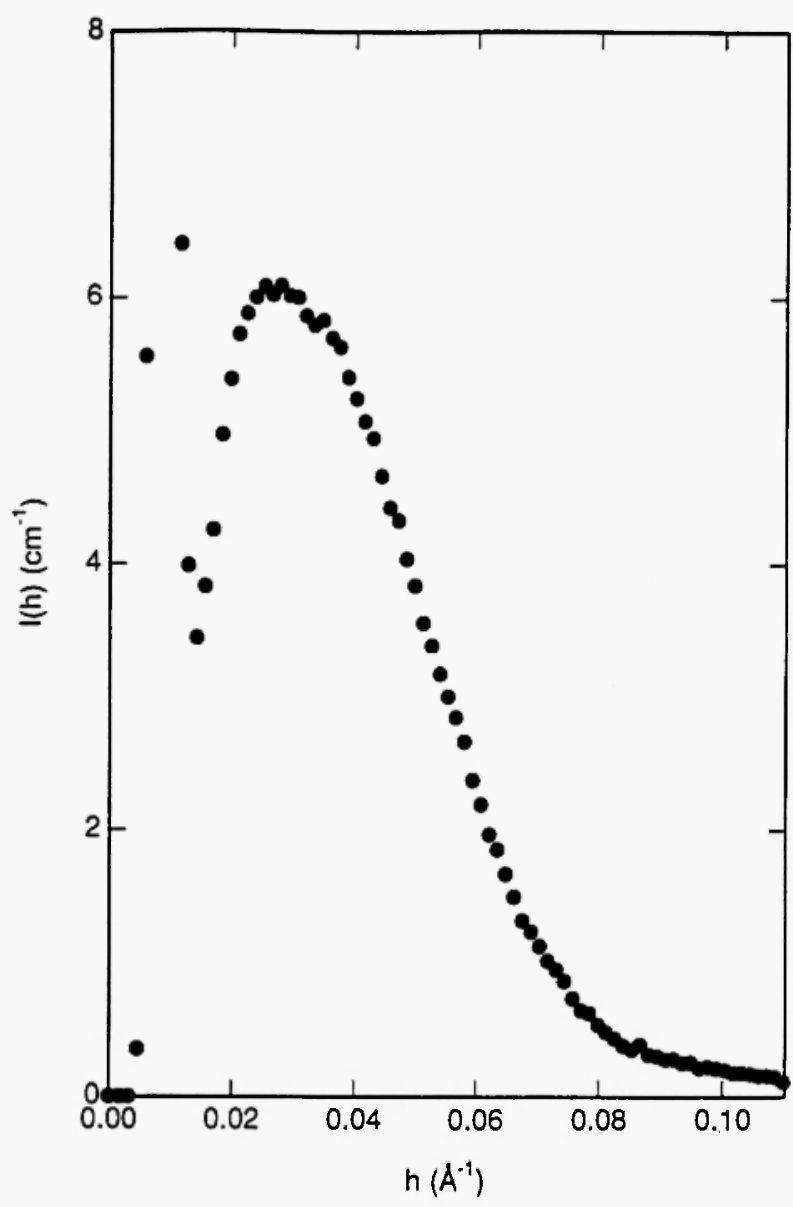

Fig. 7: Scattered intensity for Lupolen standard.

The Guinier law /1/

$I(q) \equiv I(0) \exp \left(-\frac{q^{2} R_{g}{ }^{2}}{3}\right), \quad$ for $q \leqq \frac{1}{R_{8}}$

yields $R_{8}=7.3 \pm 0.3 \AA$ for the silica sol of HS-40 as shown in Fig. 8, where $R_{g}$ is the radius of gyration of scattering particles. To check this result, we performed electron micrograph observation for the same sample. 


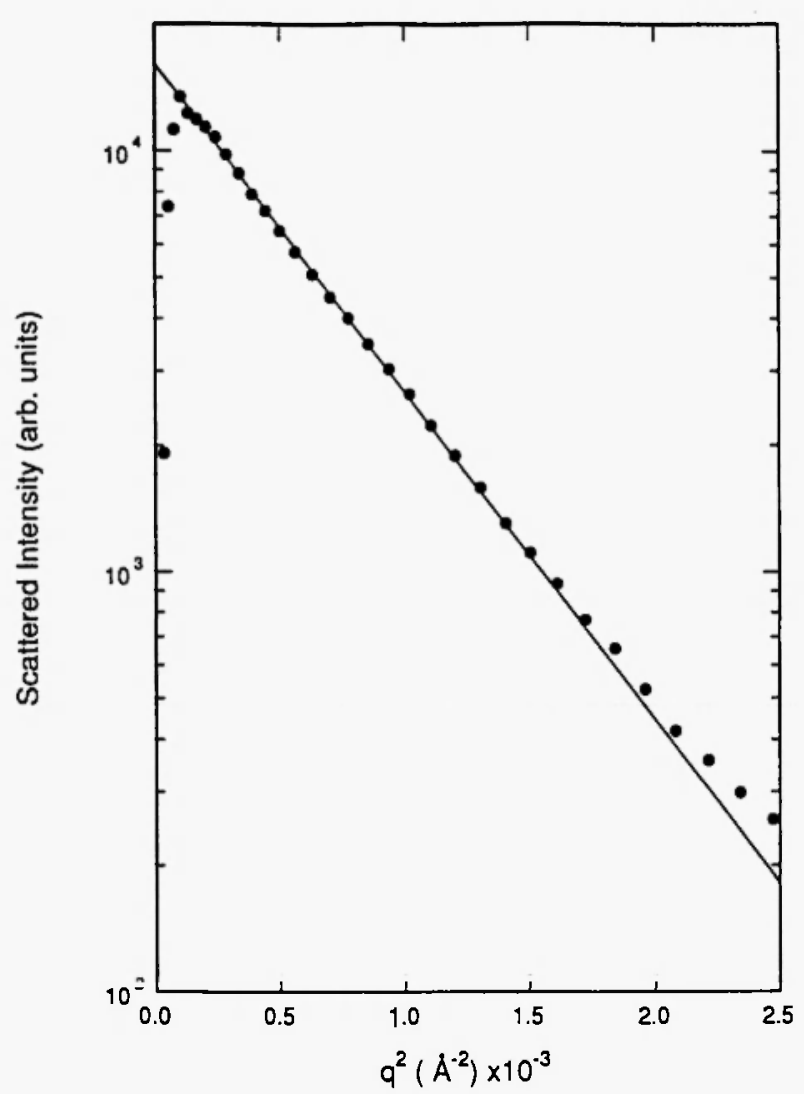

Fig. 8: Guinier plot for $0.95 \mathrm{wt} . \% \mathrm{SiO}_{2}$ (Ludox HS40).

From the electron micrographs, a value $72.7 \pm 0.9 \AA$ was obtained for the $z$-averaged radius of gyration of silica particles. The $R_{8}$ value obtained from the SAXS measurement was in fair agreement with that of TEM observation within experimental error.

\section{THE STRUCTURE OF COLLOIDAL SUSPENSION}

Colloidal suspension LUDOX HS-40 (DuPont) consists of silica spheres about $188 \AA$ in diameter determined using Eq. (8); it is considered to have both inter- and intra-particle structure on length scales being well suited for measurement by the constructed SAXS instrument. The SAXS measurements were performed to study the structure and interactions between particles in the silica sols and the dependence on silica concentration. The sols of silica concentrations $0.950,4.58$, $15.4,19.1,24.4,29.9,34.2$ and $39.1 \mathrm{wt} \%$ were pre- pared by diluting silica suspension with distilled water. Concentrations were determined by evaporating the water in a given volume of suspension and weighing the remaining silica. The scattered intensities were measured for the silica suspensions of various concentrations and are plotted in Fig. 9 in absolute units.

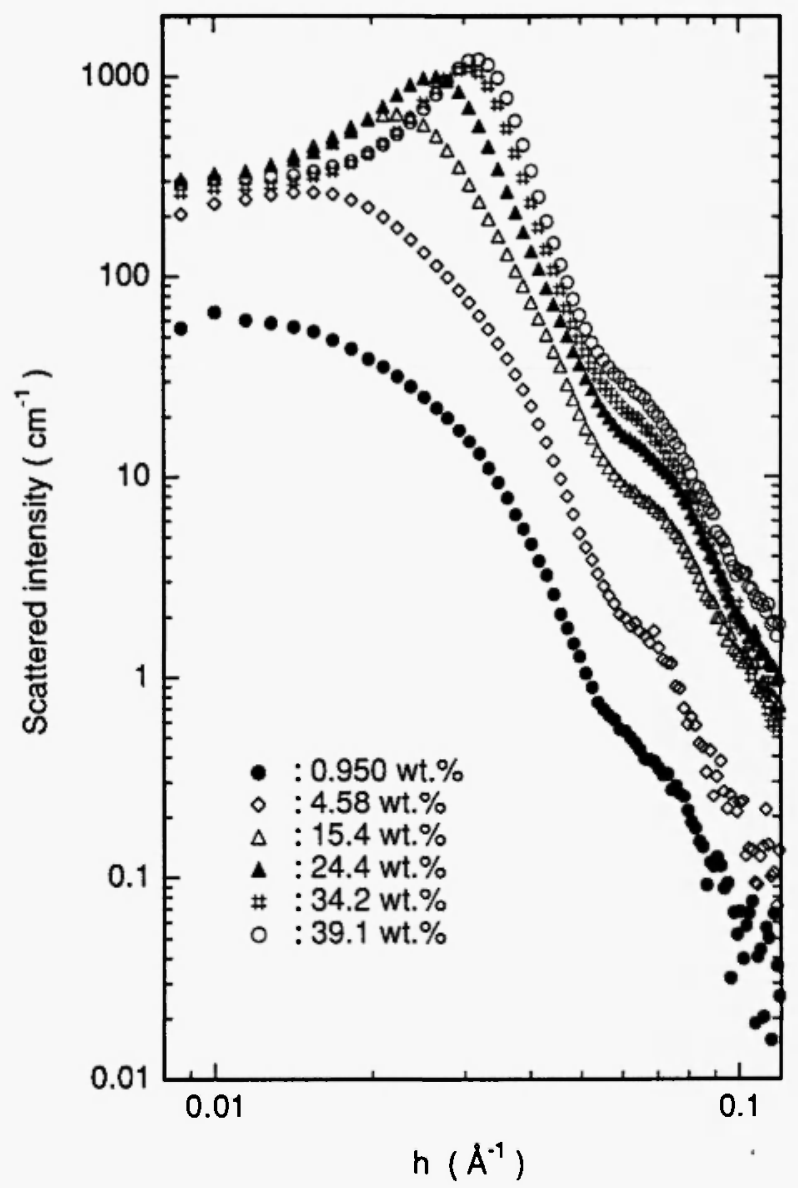

Fig 9: Scattered intensities for silica colloidal suspensions (Ludox HS-40) at silica concentrations $0.950,4.58,15.4,24.4,34.2$ and 29.1 wt.\%.

We consider the colloidal dispersion as a "macrofluid", in which the colloidal particles exist in a structureless solvent and interact with each other via potentials, and interpret the scattered intensities in terms of a product

$I_{\text {mod al }}(q)=n P(q) S(q)$,

where $n$ is the particle number density, $P(q)$ is the 
particle form factor and $S(q)$ is the interparticle structure factor (e.g. /II/). Equation (9) is given for a monodisperse population of spherical scatterers. An oscillatory behavior about the Porod line in the scattered intensities observed near $q=0.7 \AA^{-1}$ in Fig. 9 indicates that the Ludox suspensions can be regarded as being monodisperse systems approximately. The single particle form factor $P(q)$ for spheres of radius $R$ is given by

$P(\bar{y})-\left\{\frac{3(\sin q R-q R \cos q R)}{(q R)^{3}}\right\}^{2}$.

The structure factor $S(q)$ depends on the interparticle scattering. The Percus-Yevick approximation is known to provide a good description of the properties of fluids in which the molecular interactions are short-ranged /12/. However, it is known in the colloidal suspension Ludox HS-40 that hydroxide ions in the alkaline solvent link to silanols being spread over the surface of a colloidal silica particle and give the silica particle a net minus charge. The silica particle is considered not to settle out of the sols due to the interparticle repulsion forces originating from the net minus charge.

In the mean spherical approximation, closed analytic forms for the structure factor $S(q)$ have been obtained by solving the Ornstein-Zernike equation for a macroion solution comprising identical spherical hardcores of diameter $\sigma$ interacting between them through the repulsive screened Coulomb pair potential $U(r)$ given by the Yukawa form /13/

$\mathrm{U}(\mathrm{r})=\frac{\pi \varepsilon_{0} \varepsilon \sigma^{2} \psi_{0}^{2} \exp [-\kappa(\mathrm{r}-\sigma)]}{\mathrm{r}}, \quad \mathrm{r}>\sigma$,

where $r$ is the inter ionic center-to-center distance, $\psi_{0}$ is the surface potential, $\varepsilon$ is the dielectric constant of the solvent medium, $\varepsilon_{0}$ is the permittivity of free space, and $\kappa$ is the Debye-Hückel inverse screening length.

Non-linear least squares fits were performed for model scattered intensity in Eq. (9), representing $S(q)$ by the equations given by Hayter and Penfold using the mean spherical approximation/13/ and determined values of $\psi_{0}, k$ and the volume fraction $\eta$. The diameter of core $\sigma$ was used as a fixed value given by a constant $2 \mathrm{R}$ throughout the fitting procedures, where a constant value $R=94 \AA$ given in Eq. (7), radius of the silica particle, is calculated from the radius of gyration obtained by the Guinier law (8).

The fitting procedures were made by using the program system SALS /14/. The qualities of the fits were checked using the value $S$ of standard deviation of fit, that is, a criterion $S \sim 1$ must be met to obtain a good fit $/ 14 \%$. The rather good fits of the scattered intensity near the maxima to the model intensity (9) could be obtained with the $S$ values better than 3 for all the solutions except for 34.2 and 39.1 wt.\% solutions. Only the fitting procedures for 34.2 and $29.1 \mathrm{wt} \%$ solutions give the $S$ values of 6.4 and 79.5 , respectively. The structural features of silica sols can be described well to a concentration of $30 \mathrm{wt} . \%$ within the framework of the mean spherical approximation, while those of concentrated silica sols (> $34 \mathrm{wt} . \%$ ) appear not to be described well by the mean spherical approximation, especially for a concentration of $39.1 \mathrm{wt} \%$. Fitted $I_{\text {model }}(q)$ and $S(q)$ curves are plotted in Fig. 10 as an example, together with the particle form factor $P(q)$ for $R=94 \AA$ and the experimental scattered intensity for the $19.1 \mathrm{wt}$. solution. It should be noted in the figure that the first peak in $S(q)$ does not correspond to the observed peak.

The mean spherical approximation fails to provide good fits to the tail of the experimental SAXS patterns and the second peak in $S(q)$ cannot be identified in SAXS patterns clearly, as shown in Fig. 10. This disagreement may be attributable at least partly to the fact that the silica sol is not a monodisperse system, because the model equation (9) is given for a monodisperse population of spherical scatterers.

Finally, structural results from the fits for the experimental SAXS data re presented. Fitted interparticle structure factors $S(q)$ are illustrated for various silica particle concentrations in Fig. 11. Values for the volume fraction $\eta$, the surface potential $\psi_{0}$ and the Debye-Huckel screening length $\mathrm{K}^{-1}$ obtained from the fits to the experimental SAXS data are tabulated in Table 1, together with the volume fraction $V_{f}$ obtained from gravimetric data.

\section{CONCLUSIONS}

A pinhole SAXS camera utilizing the imaging plate 


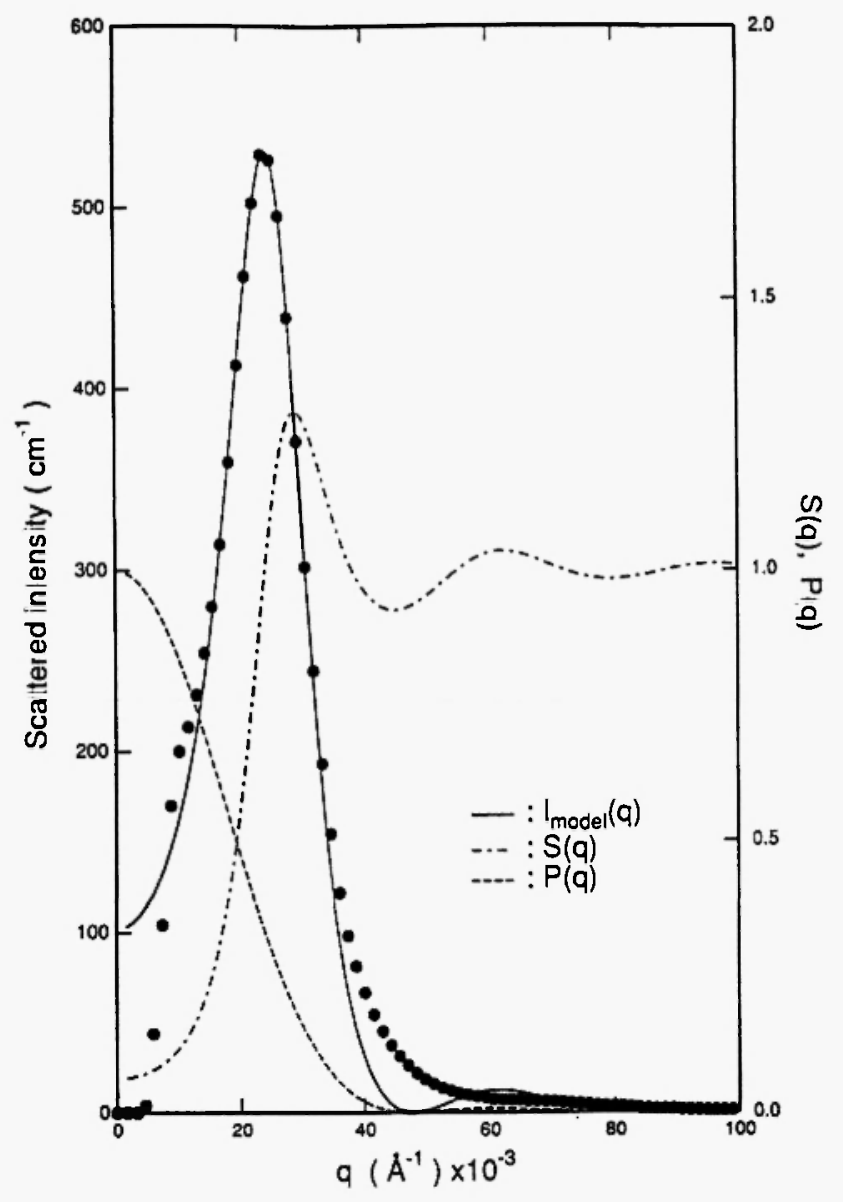

was designed and constructed. The geometry of the instrument can be adjusted readily, so as to be suited for SAXS measurements corresponding to a length scale of the structural inhomogeneities to be investigated. Mounting a rotating-node $\mathrm{X}$-ray tube on a goniometer makes it easy to choose the wavelength of the X-rays in combination with a monochromator crystal. The choice of different wavelengths of the incident $\mathrm{X}$-rays makes the sample preparation much easier and extends the species of samples especially for heavy metal specimens.

The utilization of a two-dimensional positionsensitive detector, the imaging plate, brings not only the direct observation of the anisotropic scattering patterns from the entities such as fibers and the fine structures in the scattering pattern like an oscillation about the $\mathrm{h}^{-4}$ line but also an increase in a signal-to-

Fig. 10: Fitted $I_{\text {model }}(q)$ and $S(q)$ curves and particle form factor $P(q)$ for $R=94 \AA$ versus scattering vector $q$, together with the experimental scattered intensity for $19.1 \mathrm{wt} . \%$ solution.

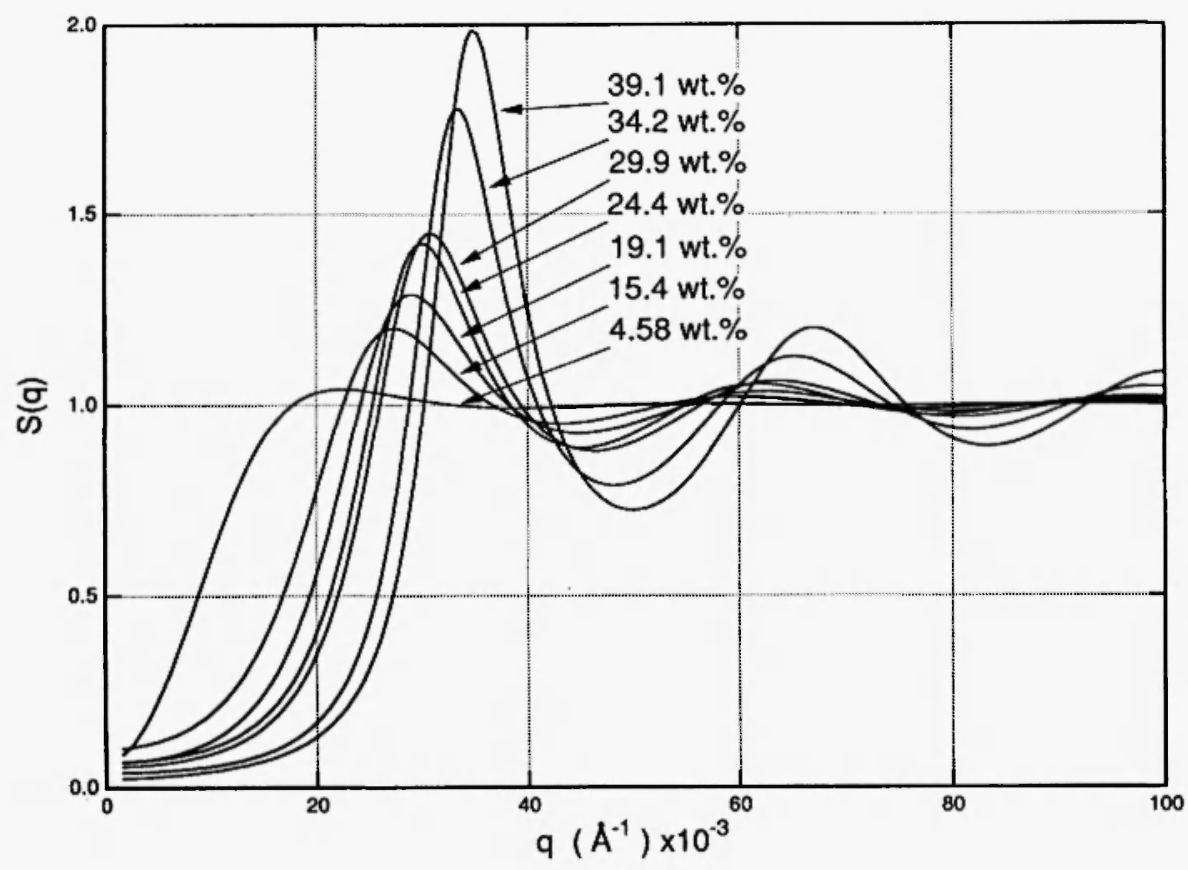

Fig. 11: Fitted interparticle structure factors $S(q)$ at silica concentrations 4.58, 15.4, 19.1, 24.4, 29.9, 34.2 and 39.1 wt.\%. 
Table 1

Volume fraction $\eta$, Surface potential $\psi_{0}$ and the Debye-Huckel screening length $\boldsymbol{\kappa}^{-1}$ obtained from the fits to the experimental SAXS data, and the volume fraction $V_{\mathrm{f}}$ obtained from gravimetric data.

\begin{tabular}{|c|c|c|c|c|}
\hline sample (wt.\%) & vol. fraction $V_{\mathrm{f}} \%$ & vol. fraction $\eta \%$ & $\begin{array}{c}\text { screening length } \\
(\AA)\end{array}$ & $\begin{array}{c}\text { surface potential } \\
\psi_{0}(\mathrm{mV})\end{array}$ \\
\hline 4.58 & 2.25 & $4.6 \pm 0.2$ & $320 \pm 239$ & 16 \\
\hline 15.4 & 8.03 & $14.9 \pm 0.5$ & $65 \pm 13$ & 17 \\
\hline 19.1 & 10.2 & $20.0 \pm 0.4$ & $61 \pm 11$ & 18 \\
\hline 24.4 & 13.4 & $23.3 \pm 0.1$ & $38 \pm 2$ & 18 \\
\hline 29.9 & 17.0 & $25.6 \pm 0.1$ & $46 \pm 2$ & 17 \\
\hline 34.2 & 20.0 & $35.0 \pm 0.1$ & $79 \pm 2$ & 16 \\
\hline 39.1 & 23.6 & $39.1 \pm 0.4$ & $104 \pm 2$ & 15 \\
\hline
\end{tabular}

noise ratio of scattered intensity by orders of magnitude, especially in the Porod region, by the circular average of two-dimensional data.

The setting of the imaging plate in an evacuated chamber makes it unnecessary to use the large size exit window. The absorption of the scattered $\mathrm{X}$-rays by the exit window can be avoided. Further, this settling promises SAXS measurements free from any correction for the non-uniformity of the thickness of the window and the shadowing effect of the grid supporting the exit window.

The smearing effects of this pinhole SAXS instrument are negligible and the raw data can be used for further analysis without smearing. A comparison of the measured values for a differential cross section and the size of structural inhomogeneities with the previous data and the results of TEM observation could make sure that structural measurements can be made using the SAXS instrument with sufficient accuracy.

One of the important features of the small-angle scattering method is its potential to make a structural investigation of a liquid specimen like a colloidal suspension. A good fit of the experimental SAXS pattern for the silica sols with various silica concentrations can be obtained near the maxima to the model function calculated using the mean spherical approximation.

\section{ACKNOWLEDGMENTS}

The authors would like to thank members of the IMR machine shop for fabricating all the SAXS instrument. The authors also wish to express their thanks to T. Konno for the TEM measurement of silica spheres, $K$. Suzuya for providing bovine collagen and members of the IMR chemical analysis division for the determination of the concentrations of silica sols. This research was partly supported by the Research Program of Pseudo-Molecular Science accepted in the Center for Interdisciplinary Research, Tohoku University.

\section{REFERENCES}

1. A. Guinier and G. Fournet, Small-Angle Scattering of $X$-Rays, John Wiley \& Sons, New York/Chapman \& Hall, London, 1955.

2. R.W. Hendricks, J. Appl. Cryst., 11, 15-30 (1978).

3. G.D. Wignall, J.S. Lin and S. Spooner, J. Appl. Cryst., 23, 241-245 (1990).

4. J. Miyahara, K. Takahashi, Y. Amemiya, N. Kamiya and $\mathrm{Y}$. Satow, Nucl. Instr. Methods, A246, $572-578$ (1986).

5. NOVA*GKS Reference Guide, C Language Binding, Version 2.2, Nova Graphics International 
Corporation, 1988.

6. J. Schelten and R.W. Hendricks, J. Appl. Cryst., 8, 421-429 (1975).

7. R.W. Hendricks and P.W. Schmidt, Acta Phys. Austriaca, 26, 97-122 (1967).

8. P.B. Moore, J. Appl. Cryst., 13, 168-175 (1980).

9. L.A. Feigin and D.I. Sverbun, Structure Analysis. by. Small-Angle $X$-Ray and Neutron Scattering, Plenum Press, New York, 1987.

10. T.R. Russell, J.S. Lin, S. Spooner and G.D. Wignall, J. Appl. Cryst., 21, 629-638 (1988).

11. E.W. Kaler, in: Modern Aspects of Small-Angle Scattering, H. Brumberger (Ed.), Kluwer
Academic Publishers, The Netherlands, 1995; pp. 329-353.

12. S.A. Rice and P. Gray, The Statistical Mechanics of Simple Liquids. An Introduction to the Theory, of Equilibrium and Non-Equilibrium Phenomena, Interscience Publishers, New York, 1965.

13. J.B. Hayter and J. Penfold, Mol. Phys., 42, 109118 (1981).

14. T. Nakagawa and Y. Oyanagi, in: Recent Developments in Statistical Interference and Data Analysis, K. Matusita (Ed.), North Holland Publishing Company, Amsterdam, 1980; pp. 221225. 